Supporting Information belonging to the manuscript:

\title{
The Effect of the Metal Fragment in the Thermal Cycloaddition between Alkynylmetal(0) Fischer Carbene Complexes and Nitrones
}

\author{
Israel Fernández, ${ }^{*}$ Miguel A. Sierra, and Fernando P. Cossío.
}

Departamento de Química Orgánica, Facultad de Química, Universidad Complutense, 28040-

Madrid, Spain. Kimika Fakultatea. Euskal Herriko Unibertsitatea. P.K. 1072, 28080-San Sebastián

Donostia, Spain

e-mail: israel@quim.ucm.es

Cartesian coordinates (in $\AA$ ) and total energies (in a. u., non corrected zero-point vibrational energies included) of all the stationary points discussed in the text. All calculations have been performed at the B3LYP/LANL2DZ\&6-31+G(d) $+\Delta$ ZPVE level of theory.

Alkynylcarbene complexes and alkynylesters $1 \mathrm{a}-\mathrm{g}$.

1a: $E=-882.87126$

$\begin{array}{ll}24 & -0.609254000 \\ 6 & -2.517031000 \\ 6 & -0.570542000 \\ 6 & -0.597535000 \\ 8 & -0.547374000 \\ 8 & -3.670860000 \\ 8 & -0.574156000 \\ 6 & -0.597814000 \\ 6 & -0.572225000 \\ 8 & -0.575422000 \\ 8 & -0.551049000 \\ 6 & 1.406399000 \\ 8 & 2.072050000 \\ 6 & 3.521228000 \\ 6 & 2.209881000 \\ 6 & 2.800329000 \\ 1 & 3.765049000 \\ 1 & 3.914535000 \\ 1 & 3.914101000 \\ 1 & 3.306768000\end{array}$
$-0.014483000$
0.016080000
1. 335309000
$-1.372877000$
2. 152748000
0.029740000
$-2.193552000$
$-1.388310000$
1. 350601000
$-2.218235000$
2. 177224000
$-0.068656000$
$-1.218709000$
$-1.271774000$
1. 110107000
2.173886000
$-2.333941000$
$-0.785514000$
$-0.787762000$
3.114887000

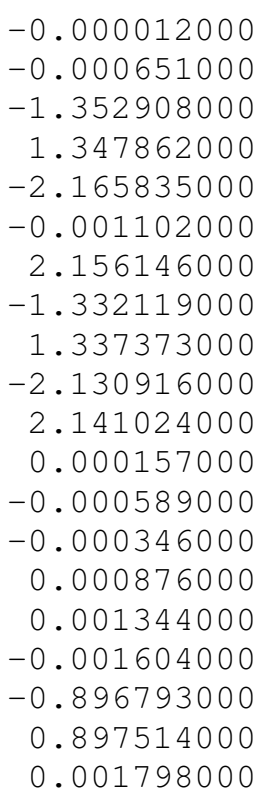

0.001798000 


$\begin{array}{lr}24 & 0.837056000 \\ 6 & 2.529759000 \\ 6 & 0.205958000 \\ 6 & 1.414956000 \\ 8 & -0.183353000 \\ 8 & 3.559006000 \\ 8 & 1.751063000 \\ 6 & 1.435894000 \\ 6 & 0.189400000 \\ 8 & 1.786382000 \\ 8 & -0.208064000 \\ 6 & -0.962970000 \\ 8 & -1.039731000 \\ 6 & -2.311658000 \\ 6 & -2.202500000 \\ 6 & -3.194211000 \\ 1 & -2.057625000 \\ 1 & -2.880910000 \\ 1 & -2.880369000 \\ 6 & -4.366543000 \\ 1 & -4.353995000 \\ 1 & -5.299884000 \\ 1 & -4.367496000\end{array}$

1c: $E=-903.74210$

$\begin{array}{lr}74 & -0.634811000 \\ 6 & -2.501390000 \\ 6 & 0.012130000 \\ 6 & -1.235321000 \\ 8 & 0.389676000 \\ 8 & -3.551741000 \\ 8 & -1.562545000 \\ 6 & -1.230692000 \\ 6 & 0.007293000 \\ 8 & -1.555158000 \\ 8 & 0.382326000 \\ 6 & 1.317489000 \\ 8 & 1.446700000 \\ 6 & 2.745788000 \\ 6 & 2.527925000 \\ 6 & 3.492707000 \\ 1 & 2.537583000 \\ 1 & 3.304014000 \\ 1 & 3.304226000 \\ 6 & 4.635737000 \\ 1 & 4.598631000 \\ 1 & 5.587303000 \\ 1 & 4.620187000\end{array}$

1d: $E=-1173.56798$
$-0.159399000$

$-1.026850000$

$-1.334235000$

1.051809000

$-2.047429000$

$-1.551079000$

1.790254000

1.088876000

$-1.372925000$

1.848489000

$-2.110100000$

0.780014000

2.108061000

2.801078000

0.086262000

$-0.624837000$

3.861072000

2.541370000

2.542764000

$-1.488355000$

$-2.146405000$

$-0.913624000$

$-2.130207000$

$-0.100830000$

$-0.974446000$

$-1.429548000$

1.257007000

$-2.178510000$

$-1.459081000$

2.025315000

1. 244694000

$-1.417679000$

2. 006194000

$-2.160022000$

0.827669000

2.154988000

2.792479000

0.083503000

$-0.663813000$

3. 862452000

2.510659000

2. 509816000

$-1.566301000$

$-2.230565000$

$-1.022694000$

$-2.200688000$
0.000051000

$-0.000368000$

$-1.364095000$

1. 362502000

$-2.183934000$

$-0.000770000$

2. 181371000

$-1.319258000$

1. 321515000

$-2.112268000$

2. 115872000

$-0.000411000$

$-0.001124000$

$-0.001275000$

$-0.000405000$

0.000189000

$-0.002176000$

$-0.897824000$

0.896025000

0.002166000

0.880249000

0.013947000

$-0.887979000$
$-0.000003000$

$-0.000614000$

1. 432665000

$-1.433437000$

2. 227885000

$-0.001039000$

$-2.230176000$

1. 447010000

$-1.445949000$

2. 251370000

$-2.248499000$

0.000037000

$-0.000568000$

$-0.000707000$

0.000392000

0.000457000

$-0.001237000$

0.896352000

$-0.897364000$

0.001034000

$-0.871580000$

$-0.019370000$

0.896304000
$-0.239514000$

$-1.387587000$

$-1.191821000$

0.757647000

$-1.765927000$

$-2.077805000$

1. 373499000

0.971158000

$$
\begin{array}{r}
0.000673000 \\
0.000503000 \\
-1.478336000 \\
1.475774000 \\
-2.366425000 \\
0.000192000 \\
2.360082000 \\
-1.191662000
\end{array}
$$




$\begin{array}{ll}6 & 0.179082000 \\ 8 & 2.488658000 \\ 8 & -0.361405000 \\ 6 & -0.512822000 \\ 8 & -0.369801000 \\ 6 & -1.510694000 \\ 6 & -1.851168000 \\ 6 & -2.960431000 \\ 1 & -1.084120000 \\ 1 & -2.116988000 \\ 1 & -2.110740000 \\ 14 & -4.610630000 \\ 1 & -4.479145000 \\ 1 & -5.512925000 \\ 1 & -5.134174000\end{array}$

1e: $E=-1113.86483$
-1.424312000
1.705463000

$-2.146830000$

0.997431000

2. 319387000

3. 214159000

0.515539000

$-0.008539000$

4. 217011000

3. 046261000

3.058592000

$-0.817146000$

$-2.222307000$

$-0.070754000$

$-0.764772000$
1.196619000
-1.907217000
1.914830000
-0.003139000
-0.007241000
-0.009537000
-0.003319000
-0.002567000
-0.017808000
-0.903453000
0.890821000
0.007539000
-0.442433000
-0.902602000
1.393999000
$-0.332439000$

$-1.700232000$

$-1.254738000$

0.639120000

$-1.817795000$

$-2.529947000$

1.234318000

0.639174000

$-1.254831000$

1.234385000

$-1.817957000$

1.153938000

2.433205000

3. 508178000

0.908594000

0.562628000

4.424497000

3. 453325000

3. 453430000

0.140541000

$-1.237770000$

1.086107000

$-1.963470000$

2.145411000

$-1.653321000$

0.659942000

$-2.714558000$

1.392192000

$-0.708149000$

$-1.037052000$
0.000028000

0.000127000

$-1.341750000$

1. 342865000

$-2.146452000$

0.000188000

2.150669000

$-1.342564000$

1. 341545000

$-2.150225000$

2. 146085000

$-0.000075000$

$-0.000156000$

$-0.000229000$

$-0.000054000$

$-0.000041000$

$-0.000278000$

$-0.897396000$

0.896931000

$-0.000018000$

$-0.000243000$

0.000229000

$-0.000431000$

0.000401000

$-0.000216000$

0.000250000

$-0.000387000$

0.000439000

0.000029000

0.000047000

1f: $E=-344.44688$

$-0.069989000$

0.089188000

0.363733000

1.478742000

$-0.776990000$

$-0.602580000$

$-1.612794000$

$-0.055446000$

$-0.054944000$

$-0.266958000$

0.685945000
-0.000374000
-0.000140000
-0.000048000
0.000055000
0.000008000
0.000041000
0.000340000
-0.892109000
0.891911000
-0.000276000
-0.114365000 
1g: $E=-1967.72530$

$\begin{array}{lr}6 & -2.904115000 \\ 6 & -2.167352000 \\ 6 & -1.208570000 \\ 8 & 0.031446000 \\ 8 & -1.698095000 \\ 6 & -0.752044000 \\ 6 & -3.763991000 \\ 13 & 1.198616000 \\ 17 & 0.685648000 \\ 17 & 0.686774000 \\ 17 & 3.104391000 \\ 1 & -1.377835000 \\ 1 & -0.129524000 \\ 1 & -0.129498000 \\ 1 & -3.559612000 \\ 1 & -4.822785000 \\ 1 & -3.558960000\end{array}$

\section{Nitrones $3 a, b$}

3a: $E=-209.05240$

$\begin{array}{lr}6 & -1.087536000 \\ 7 & -0.124526000 \\ 8 & -0.287544000 \\ 6 & 1.292763000 \\ 1 & -0.844629000 \\ 1 & -2.105380000 \\ 1 & 1.762812000 \\ 1 & 1.365076000 \\ 1 & 1.762787000\end{array}$
$-0.824833000$
0.057590000
1.321829000
$-0.383034000$
$-1.877680000$
$-0.458742000$
0.039042000
$-1.472106000$
0.038931000

3b: $E=-169.76304$

$\begin{array}{rr}6 & -1.131228000 \\ 7 & 0.056324000 \\ 8 & 1.187955000 \\ 1 & -1.989337000 \\ 1 & -1.208344000 \\ 1 & 0.087135000\end{array}$
$-0.193143000$
0.345910000
$-0.229167000$
0.466004000
$-1.272508000$
1. 377326000
$-0.000019000$
0.000017000
$-0.000011000$
$-0.000011000$
$-0.000012000$
$-0.000043000$
$-0.000058000$
0.000012000
$-1.798463000$
1.799448000
$-0.000924000$
$-0.000084000$
0.895035000
$-0.895100000$
0.885821000
$-0.000507000$
$-0.885493000$

\section{Transition States Pathway A TSa(A)-TSg(A)}

TSa (A) : $E=-1091.90652$

$\begin{array}{lrrr}6 & 2.549151000 & 1.134691000 & -0.588795000 \\ 24 & 1.365671000 & -0.210537000 & 0.062929000 \\ 6 & 2.893511000 & -1.171168000 & 0.573619000 \\ 8 & 3.834895000 & -1.764666000 & 0.895050000 \\ 8 & 3.338817000 & 1.887527000 & -0.972641000 \\ 6 & 1.242752000 & 0.618052000 & 1.763556000 \\ 8 & 1.142511000 & 1.107374000 & 2.808856000 \\ 6 & 0.317784000 & -1.646110000 & 0.713851000 \\ 8 & -0.294630000 & -2.535518000 & 1.134307000 \\ 6 & 1.354641000 & -1.001618000 & -1.678324000\end{array}$


$-1.479115000$

0.810299000

0.075792000

2. 126072000

3. 132077000

$-0.630011000$

$-0.321192000$

$-0.312684000$

$-1.125134000$

0.712697000

3.253045000

4.051786000

2.891950000

$-1.133110000$

0.430930000

$-1.119787000$

1.298564000

0.215099000

1. 348331000
-2.728122000
-0.540931000
-0.996393000
-0.583602000
-0.262811000
-1.556830000
0.439171000
1.357628000
-0.599916000
0.301334000
-1.087942000
-0.134874000
0.658495000
-2.459492000
2.143198000
1.377322000
1.219883000
0.097073000
-0.545026000

TSb (A) : $E=-1091.91122$

$\begin{array}{lr}6 & 2.469784000 \\ 24 & 1.237313000 \\ 6 & 2.688868000 \\ 8 & 3.583044000 \\ 8 & 3.285112000 \\ 6 & 1.514212000 \\ 8 & 1.654725000 \\ 6 & 0.144527000 \\ 8 & -0.486387000 \\ 6 & 0.814524000 \\ 8 & 0.538421000 \\ 6 & -0.485009000 \\ 6 & -1.725889000 \\ 8 & -0.606710000 \\ 6 & 0.523987000 \\ 6 & -2.727754000 \\ 6 & -3.331450000 \\ 1 & 1.084509000 \\ 1 & 0.102477000 \\ 1 & 1.172649000 \\ 1 & -2.602173000 \\ 1 & -4.230347000 \\ 1 & -3.616799000 \\ 7 & -3.839738000 \\ 6 & -2.716008000 \\ 8 & -4.052870000 \\ 1 & -4.404493000 \\ 1 & -2.511709000 \\ 1 & -2.152920000\end{array}$

0.514038000

$-0.237934000$

$-1.213833000$

$-1.813890000$

0.899413000

1.176564000

2.036389000

$-1.115107000$

$-1.665126000$

$-1.587840000$

$-2.395422000$

0.794603000

0.179830000

2. 008920000

2.859783000

$-0.555011000$

$-1.606258000$

2.512679000

3. 845963000

2. 903235000

$-1.933590000$

$-1.244276000$

$-2.472833000$

0.800295000

1.028868000

$-0.341408000$

1.582902000

2.042590000

0.186856000
$-1.112163000$

0.131763000

0.813658000

1. 239460000

$-1.836421000$

1.365782000

2. 128672000

1. 407502000

2. 208081000

$-1.150117000$

$-1.927490000$

$-0.623590000$

$-0.579683000$

$-1.193379000$

$-1.423730000$

$-0.814169000$

$-1.661295000$

$-2.294953000$

$-1.628529000$

$-0.547796000$

$-2.408924000$

$-2.172927000$

$-1.053268000$

1.006987000

1. 632057000

0.398063000

0.669804000

1.958035000

2. 005935000

TSc (A): $E=-1073.48106$

$\begin{array}{lr}6 & 2.236962000 \\ 74 & 0.970463000 \\ 6 & 2.598508000 \\ 8 & 3.525016000 \\ 8 & 3.011245000 \\ 6 & 1.290730000 \\ 8 & 1.457750000 \\ 6 & -0.155904000 \\ 8 & -0.766413000\end{array}$

0.766155000 $-0.160774000$

$-1.189862000$

$-1.775050000$

1.236298000

1.310521000

2. 139698000

$-1.200804000$

$-1.803195000$
-1.249237000
0.079665000
0.737884000
1.117102000
-1.970906000
1.472961000
2.265537000
1.437529000
2.217945000 


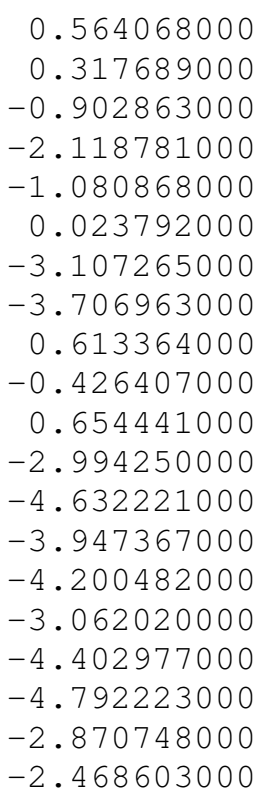
$-1.601903000$
$-2.404997000$
0.880944000
0.216200000
2. 110641000
2.999434000
$-0.537268000$
$-1.576179000$
2. 674230000
3. 971787000
3. 059649000
$-1.854995000$
$-1.223471000$
$-2.471542000$
0.728369000
0.965654000
$-0.398704000$
1. 507258000
1. 973751000
0.127151000

-1.337763000
-2.131164000
-0.638192000
-0.590974000
-1.164179000
-1.373414000
-0.822105000
-1.687157000
-2.234003000
-1.582892000
-0.484191000
-2.469610000
-2.156447000
-1.101828000
1.072032000
1.667368000
0.432640000
0.774912000
2.017668000
1.999776000

TSd (A) : $E=-1131.20234$

$\begin{array}{lr}6 & 2.684571000 \\ 24 & 1.491126000 \\ 6 & 3.015380000 \\ 8 & 3.955718000 \\ 8 & 3.486826000 \\ 6 & 1.508529000 \\ 8 & 1.484498000 \\ 6 & 0.457380000 \\ 8 & -0.137570000 \\ 6 & 1.299347000 \\ 8 & 1.158765000 \\ 6 & -0.321708000 \\ 6 & -1.470855000 \\ 8 & -0.560702000 \\ 6 & 0.476572000 \\ 6 & -2.403286000 \\ 6 & -2.854827000 \\ 1 & 1.124364000 \\ 1 & -0.041401000 \\ 1 & 1.064690000 \\ 1 & -2.070710000 \\ 1 & -3.776094000 \\ 1 & -3.050939000 \\ 7 & -3.685863000 \\ 6 & -2.635864000 \\ 8 & -3.722525000 \\ 6 & -4.651454000 \\ 1 & -2.536383000 \\ 1 & -1.989549000 \\ 1 & -4.594265000 \\ 1 & -5.648623000 \\ 1 & -4.409848000\end{array}$

0.681233000

$-0.156254000$

$-0.896856000$

$-1.353103000$

1.127667000

1. 348549000

2.263368000

$-1.099531000$

$-1.684197000$

$-1.607616000$

$-2.478913000$

0.611068000

$-0.142233000$

1. 767597000

2.719825000

$-0.988474000$

$-2.164893000$

2. 367251000

3. 626994000

2.922273000

$-2.451350000$

$-1.953329000$

$-3.015842000$

0.367192000

0.714416000

$-0.840150000$

1. 318804000

1. 745185000

$-0.062064000$

2.263971000

0.885738000

1.467013000
-1.064976000
0.161433000
0.961681000
1.461920000
-1.769086000
1.313907000
2.024500000
1.436174000
2.240760000
-1.062660000
-1.807328000
-0.726137000
-0.673433000
-1.385884000
-1.645528000
-0.826172000
-1.606698000
-2.451867000
-1.964290000
-0.748634000
-2.313899000
-2.161694000
-0.943355000
0.860083000
1.547189000
0.321291000
0.300569000
1.865393000
1.927307000
0.843492000
0.396059000
-0.756359000

TSe (A): $E=-1112.77220$

$\begin{array}{ll}6 & 2.403924000 \\ 74 & 1.176119000 \\ 6 & 2.868525000 \\ 8 & 3.833427000 \\ 8 & 3.162326000\end{array}$

0.861016000

$-0.105715000$

$-0.884377000$

$-1.328044000$

1.361682000

$$
\begin{array}{r}
-1.232602000 \\
0.103798000 \\
0.914341000 \\
1.382067000 \\
-1.951100000
\end{array}
$$




\begin{abstract}
1. 241528000
1. 259794000

0.102250000

$-0.479476000$

1.007247000

0.892221000

$-0.775664000$

$-1.903984000$

$-1.056123000$

$-0.037123000$

$-2.831932000$

$-3.290514000$

0.626813000

$-0.570017000$

0.538744000

$-2.526307000$

$-4.232157000$

$-3.453289000$

$-4.074973000$

$-3.005625000$

$-4.114817000$

$-5.064590000$

$-2.904700000$

$-2.340913000$

$-5.003392000$

$-6.054360000$

$-4.851498000$
\end{abstract}

1.492694000

2. 394725000

$-1.167295000$

$-1.777559000$

$-1.678356000$

$-2.557166000$

0.662027000

$-0.122243000$

1. 822751000

2.796631000

$-0.977338000$

$-2.151705000$

2.459065000

3. 694036000

3.006744000

$-2.418439000$

$-1.947157000$

$-3.012995000$

0.346605000

0.697187000

$-0.855692000$

1.294746000

1.725736000

$-0.076495000$

2.233086000

0.849762000

1. 459841000
1.385589000

2.114346000

1.483957000

2.281266000

$-1.218252000$

$-1.960109000$

$-0.775543000$

$-0.708529000$

$-1.416385000$

$-1.665736000$

$-0.840018000$

$-1.620186000$

$-2.465595000$

$-1.987448000$

$-0.761515000$

$-2.356195000$

$-2.142612000$

$-0.961161000$

0.894825000

1. 550172000

0.343465000

0.372822000

1. 874958000

1. 903041000

0.927013000

0.487992000

$-0.687767000$

TSf (A) : $E=-1382.59482$

\begin{tabular}{|c|c|}
\hline 6 & 2.735523000 \\
\hline 24 & 1.632313000 \\
\hline 6 & 3.157022000 \\
\hline 8 & 4.097574000 \\
\hline 8 & 3.473472000 \\
\hline 6 & 1.993860000 \\
\hline 8 & 2.193787000 \\
\hline 6 & 0.664799000 \\
\hline 8 & 0.114821000 \\
\hline 6 & 1.147357000 \\
\hline 8 & 0.845569000 \\
\hline 6 & -0.155032000 \\
\hline 6 & -1.380784000 \\
\hline 8 & -0.342613000 \\
\hline 6 & 0.753211000 \\
\hline 6 & -2.380122000 \\
\hline 1 & 1.244378000 \\
\hline 1 & 0.301563000 \\
\hline 1 & 1.470887000 \\
\hline 1 & -2.176514000 \\
\hline 1 & -4.406299000 \\
\hline 1 & -3.418291000 \\
\hline 14 & -3.135289000 \\
\hline 7 & -3.455653000 \\
\hline 6 & -2.299492000 \\
\hline 8 & -3.603705000 \\
\hline 6 & -4.365428000 \\
\hline 1 & -2.083896000 \\
\hline 1 & -1.718487000 \\
\hline 1 & -4.213007000 \\
\hline 1 & -5.388737000 \\
\hline 1 & -4.160209000 \\
\hline
\end{tabular}

0.061955000

0.120782000

0.692862000

1.040900000

0.076121000

$-1.695952000$

$-2.804002000$

0.279320000

0.382153000

1. 912774000

2.993696000

$-0.494555000$

$-0.007660000$

$-1.339557000$

$-1.962807000$

0.772859000

$-1.242412000$

$-2.757225000$

$-2.385287000$

3. 064601000

2. 122572000

3.229461000

2. 385909000

$-1.179005000$

$-1.645422000$

0.138650000

$-1.912638000$

$-2.697041000$

$-1.047152000$

$-2.986194000$

$-1.643321000$

$-1.614562000$
1. 354405000 $-0.198870000$

$-1.133130000$

$-1.711702000$

2. 244741000

$-0.613330000$

$-0.882944000$

$-1.822325000$

$-2.836001000$

0.251665000

0.519221000

0.811562000

0.388855000

1. 843521000

2. 526988000

0.245397000

3. 185150000

3. 124403000

1. 822574000

1. 686997000

1. 506754000

$-0.402393000$

0.784425000

$-0.862383000$

$-1.265063000$

$-0.844314000$

0.022326000

$-1.116379000$

$-1.950355000$

$-0.104562000$

$-0.244455000$

1. 055602000 
$\operatorname{TSg}(\mathbf{A}): \quad E=-1382.59482$

$\begin{array}{lrrr}6 & -3.361953000 & -0.692401000 & 1.141485000 \\ 24 & -2.067091000 & -0.475484000 & -0.234865000 \\ 6 & -3.318684000 & -1.338479000 & -1.331420000 \\ 8 & -4.092116000 & -1.865162000 & -2.014260000 \\ 8 & -4.218001000 & -0.894300000 & 1.893944000 \\ 6 & -2.707895000 & 1.257685000 & -0.631816000 \\ 8 & -3.042473000 & 2.346517000 & -0.852688000 \\ 6 & -0.945450000 & -0.430381000 & -1.768959000 \\ 8 & -0.326354000 & -0.420233000 & -2.746489000 \\ 6 & -1.240216000 & -2.095440000 & 0.339124000 \\ 8 & -0.723016000 & -3.059574000 & 0.709164000 \\ 6 & -0.536061000 & 0.531008000 & 0.922180000 \\ 6 & 0.752147000 & 0.545617000 & 0.458135000 \\ 8 & -0.613907000 & 1.199957000 & 2.098111000 \\ 6 & -1.842386000 & 1.300610000 & 2.825438000 \\ 6 & 1.964568000 & 0.445067000 & 0.082940000 \\ 6 & 3.091148000 & -0.480966000 & 0.104840000 \\ 6 & 4.404313000 & -0.116756000 & -0.244185000 \\ 6 & 5.437340000 & -1.054737000 & -0.180570000 \\ 6 & 5.184133000 & -2.364507000 & 0.233401000 \\ 6 & 3.880545000 & -2.734968000 & 0.582160000 \\ 6 & 2.842949000 & -1.808046000 & 0.514483000 \\ 1 & -2.118029000 & 0.333064000 & 3.251648000 \\ 1 & -1.638547000 & 2.009688000 & 3.630708000 \\ 1 & -2.647108000 & 1.678878000 & 2.190787000 \\ 1 & 4.614055000 & 0.895082000 & -0.567408000 \\ 1 & 6.445386000 & -0.755861000 & -0.456918000 \\ 1 & 5.991441000 & -3.090590000 & 0.281270000 \\ 1 & 3.666872000 & -3.751934000 & 0.900695000 \\ 1 & 1.833031000 & -2.105108000 & 0.777374000 \\ 7 & 1.736606000 & 2.853622000 & -0.610805000 \\ 6 & 0.502394000 & 2.774834000 & -1.010193000 \\ 8 & 2.545400000 & 1.831258000 & -0.828189000 \\ 6 & 2.223390000 & 3.826823000 & 0.373610000 \\ 1 & -0.196279000 & 3.553358000 & -0.729014000 \\ 1 & 0.245938000 & 2.017353000 & -1.735706000 \\ 1 & 1.516629000 & 4.654548000 & 0.454091000 \\ 1 & 3.197812000 & 4.188593000 & 0.040899000 \\ 1 & 2.324769000 & 3.311688000 & 1.333325000\end{array}$

\section{Transition States Pathway B: TSa(B)-TSi(B)}

TSa (B) : $E=-1091.90792$

$\begin{array}{lr}6 & 2.130099000 \\ 24 & 1.195702000 \\ 6 & 2.395982000 \\ 8 & 3.133755000 \\ 8 & 2.746625000 \\ 6 & 2.310017000 \\ 8 & 2.976371000 \\ 6 & 0.364384000 \\ 8 & -0.097721000 \\ 6 & -0.007813000 \\ 8 & -0.766948000 \\ 6 & -0.232504000 \\ 6 & -1.425703000 \\ 8 & -0.160699000 \\ 6 & 0.989253000 \\ 6 & -2.439575000 \\ 1 & 1.031024000\end{array}$

\footnotetext{
0.488076000

$-0.339195000$

$-1.767502000$

$-2.652298000$

0.892715000

0.524347000

1.048580000

$-1.345306000$

$-2.011527000$

$-1.055216000$

$-1.469820000$

1.239295000

0.954946000

2. 564167000

3.161267000

0.672437000

2. 911000000
} 


\begin{abstract}
0.847096000
1. 908753000

$-2.847522000$

$-3.687058000$

$-3.136934000$

$-3.969251000$

$-3.822205000$

$-2.815272000$

$-3.092048000$

$-3.557333000$

$-4.856440000$

$-3.152772000$
\end{abstract}

-0.387478000
-0.008776000
2.648660000
-0.429620000
-1.254492000
0.803292000
-0.683445000
-2.224483000
-0.977139000
-1.717360000
-0.474933000
0.002669000

TSb (B) : $E=-1091.91465$

$\begin{array}{lr}6 & -1.931488000 \\ 24 & -1.186956000 \\ 6 & -2.560050000 \\ 8 & -3.405839000 \\ 8 & -2.409842000 \\ 6 & -2.243939000 \\ 8 & -2.904062000 \\ 6 & -0.456523000 \\ 8 & -0.028610000 \\ 6 & -0.097244000 \\ 8 & 0.594874000 \\ 6 & 0.459981000 \\ 6 & 1.712673000 \\ 8 & 0.509339000 \\ 6 & -0.649812000 \\ 6 & 2.754688000 \\ 6 & 3.478353000 \\ 1 & -0.966786000 \\ 1 & -0.327771000 \\ 1 & -1.468551000 \\ 1 & 2.926069000 \\ 1 & 3.581646000 \\ 1 & 4.483810000 \\ 7 & 3.291147000 \\ 6 & 2.678058000 \\ 8 & 3.940322000 \\ 1 & 2.974192000 \\ 1 & 2.010018000 \\ 1 & 2.998670000\end{array}$

1.184332000

$-0.282386000$

$-1.366236000$

$-2.034561000$

2. 035219000

0.092540000

0.275721000

$-1.788602000$

$-2.713294000$

$-0.666304000$

$-0.891322000$

0.878997000

0.302452000

2. 128123000

2.969911000

$-0.395207000$

$-1.346240000$

3. 268564000

3.848650000

2.476530000

$-1.474825000$

$-2.325283000$

$-0.975379000$

0.290204000

1.435500000

$-0.219455000$

$-0.384938000$

1.731905000

2. 130317000
1.061169000

0.112261000

0.787955000

1.211371000

1.683777000

$-1.435464000$

$-2.365832000$

$-0.807918000$

$-1.349848000$

1. 599306000

2.506234000

$-0.667578000$

$-0.710514000$

$-1.174940000$

$-1.241575000$

$-0.878737000$

$-1.750153000$

$-0.239702000$

$-1.803453000$

$-1.766630000$

$-2.686115000$

$-1.268526000$

$-1.980604000$

1. 480840000

1.373764000

0.456489000

2. 181090000

2. 174094000

0.610181000

TSC (B) : $E=-1073.48466$

$\begin{array}{lr}6 & -1.512263000 \\ 74 & -0.932543000 \\ 6 & -2.464951000 \\ 8 & -3.336596000 \\ 8 & -1.846520000 \\ 6 & -2.190024000 \\ 8 & -2.933216000 \\ 6 & -0.334717000 \\ 8 & 0.006795000 \\ 6 & 0.283145000 \\ 8 & 0.993225000 \\ 6 & 0.859800000 \\ 6 & 2.093561000 \\ 8 & 0.962409000 \\ 6 & -0.194325000 \\ 6 & 3.121322000\end{array}$

1.187480000

$-0.198152000$

$-1.399759000$

$-2.086725000$

1. 947431000

0.634453000

1.062858000

$-1.600196000$

$-2.391162000$

$-1.056141000$

$-1.529157000$

1.039709000

0.426802000

2. 343137000

3. 187327000

$-0.271541000$
1. 448064000

0.052080000

0.635974000

0.973033000

2. 257486000

$-1.355690000$

$-2.131788000$

$-1.337306000$

$-2.106812000$

1. 442153000

2. 234688000

$-0.602131000$

$-0.684315000$

$-0.941558000$

$-1.007681000$

$-0.923524000$ 


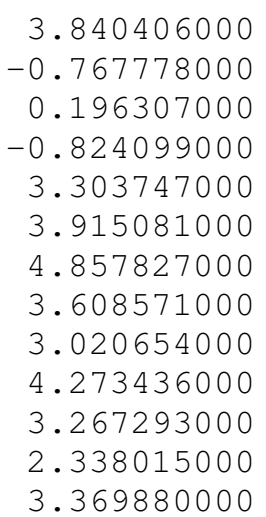

$$
\begin{array}{r}
-1.110278000 \\
3.145333000 \\
4.195110000 \\
2.904688000 \\
-1.093734000 \\
-2.148677000 \\
-0.735034000 \\
0.030594000 \\
1.191744000 \\
-0.323607000 \\
-0.742000000 \\
1.369897000 \\
1.993716000
\end{array}
$$

-1.907477000
-0.079447000
-1.160941000
-1.854627000
-2.861003000
-1.564835000
-2.068173000
1.522116000
1.589696000
0.443304000
2.099660000
2.412718000
0.954508000
2.543178000

1. 343278000

2. 595518000

3.366278000

3. 332968000

2. 121298000

2. 575338000

0.278498000

$-0.323772000$

0.426428000

$-0.170382000$

$-0.180602000$

$-1.442592000$

$-0.113313000$

1.097697000

$-2.511832000$

$-3.141272000$

1. 327027000

0.898547000

1.933307000

$-2.458732000$

$-3.364642000$

$-4.080202000$

$-3.392952000$

$-2.744002000$

$-3.853732000$

$-3.381262000$

$-2.248852000$

$-2.824852000$

$-2.986862000$

$-4.405432000$

$-2.750712000$
0.275110000

$-0.310221000$

$-1.661240000$

$-2.500260000$

0.526190000

0.883230000

1. 618320000

$-1.097761000$

$-1.638831000$

$-1.310511000$

$-1.894191000$

1.206089000

0.989059000

2. 439029000

2. 945119000

0.832719000

1. 018969000

2. 431709000

4.000359000

2.851880000

1. 578709000

0.056959000

1. 578328000

$-0.459651000$

0.229659000

0.157308000

$-1.925311000$

$-0.295341000$

1.306879000

$-2.344191000$

$-2.261542000$

$-2.212201000$
-1.233199000
0.118581000
0.460411000
0.672121000
-2.041949000
1.392401000
2.158841000
1.488571000
2.314001000
-1.184449000
-1.993019000
-0.144559000
0.347651000
-0.701499000
-1.271699000
1.012091000
2.340981000
-2.209259000
-1.469939000
-0.575009000
2.987561000
2.816851000
2.253271000
-0.960199000
-1.850719000
0.117571000
-0.875979000
-2.657959000
-1.831869000
-1.802199000
-0.703229000
-0.030299000

TSe (B) : $E=-1112.77362$

$\begin{array}{lr}6 & 2.317140000 \\ 74 & 1.067055000 \\ 6 & 2.485745000 \\ 8 & 3.292508000 \\ 8 & 3.077571000 \\ 6 & 1.907134000 \\ 8 & 2.369800000 \\ 6 & -0.053545000 \\ 8 & -0.652559000 \\ 6 & 0.131542000\end{array}$

0.551525000

$-0.206718000$

$-1.628147000$

$-2.444243000$

0.901715000

1.033661000

1.737181000

$-1.139855000$

$-1.718434000$

$-1.308861000$

$$
\begin{array}{r}
-1.362503000 \\
0.076603000 \\
0.377051000 \\
0.551095000 \\
-2.164638000 \\
1.499597000 \\
2.291246000 \\
1.525456000 \\
2.329998000 \\
-1.357739000
\end{array}
$$


$-1.901465000$

1. 322727000

1. 033406000

2.569592000

3.128393000

0.815548000

0.959877000

2.630113000

4.176490000

3.060476000

1.547402000

$-0.015633000$

1. 473565000

$-0.505988000$

0.214482000

0.086140000

$-1.970357000$

$-0.285625000$

1. 287139000

$-2.366141000$

$-2.351561000$

$-2.235483000$
-2.176857000
-0.134391000
0.367434000
-0.669940000
-1.238877000
1.022080000
2.351063000
-2.179973000
-1.429202000
-0.543463000
3.004133000
2.818461000
2.264393000
-0.960085000
-1.845607000
0.117773000
-0.882671000
-2.653020000
-1.823615000
-1.799476000
-0.740862000
-0.020232000

TSf (B) : $E=-1382.59628$

\begin{tabular}{|c|c|}
\hline 6 & 2.783844000 \\
\hline 24 & 1.495813000 \\
\hline 6 & 2.773782000 \\
\hline 8 & 3.559582000 \\
\hline 8 & 3.623991000 \\
\hline 6 & 2.105865000 \\
\hline 8 & 2.468535000 \\
\hline 6 & 0.328444000 \\
\hline 8 & -0.337899000 \\
\hline 6 & 0.784409000 \\
\hline 8 & 0.325336000 \\
\hline 6 & -0.046975000 \\
\hline 6 & -1.369432000 \\
\hline 8 & 0.031853000 \\
\hline 6 & 1.283708000 \\
\hline 6 & -2.494250000 \\
\hline 14 & -3.377934000 \\
\hline 1 & 1.677570000 \\
\hline 1 & 1.051768000 \\
\hline 1 & 2.008590000 \\
\hline 1 & -2.454742000 \\
\hline 1 & -3.762718000 \\
\hline 1 & -4.601830000 \\
\hline 7 & -3.107231000 \\
\hline 6 & -2.313046000 \\
\hline 8 & -3.720739000 \\
\hline 6 & -3.033048000 \\
\hline 1 & -1.668281000 \\
\hline 1 & -2.474248000 \\
\hline 1 & -2.507003000 \\
\hline 1 & -4.052258000 \\
\hline 1 & -2.499737000 \\
\hline
\end{tabular}

0.529147000 0.092963000

0.645901000

0.989889000

0.849261000

$-1.718119000$

$-2.813478000$

$-0.242687000$

$-0.410866000$

1. 828526000

2.886185000

$-0.587573000$

$-0.536868000$

$-1.128583000$

$-1.308898000$

$-0.821995000$

$-2.126740000$

$-0.346706000$

$-1.935019000$

$-1.813789000$

$-3.276018000$

$-1.589995000$

$-2.552398000$

1.535686000

1. 588205000

0.381454000

2. 449741000

2. 450454000

0.888667000

3. 359553000

2.679622000

1. 945895000
1.013610000 $-0.316051000$

$-1.573044000$

$-2.350998000$

1.742363000 $-0.385457000$ $-0.427788000$ $-1.784545000$ $-2.713361000$ $-0.132949000$ 0.002454000 1. 027868000 0.637229000

2. 260772000

2.934853000

0.103763000 $-0.887502000$

3. 271150000

3. 798452000

2.294183000

$-1.045799000$

$-2.216283000$

$-0.163292000$

0.586627000

1. 623579000

0.344463000

$-0.558098000$

1. 740695000

2.430942000 $-0.266580000$ $-0.874413000$ $-1.369298000$

TSg (B): $E=-1322.89082$
6
3.523844000
$-0.724985000$
0.725004000
$-0.344160000$
$24 \quad 1.981646000$
$-0.415595000$
$-1.775745000$ 


\begin{abstract}
3.788684000
4.530794000

1.386466000

0.999998000

0.638271000

$-0.104060000$

2.375161000

2.554273000

0.564213000

$-0.733389000$

0.712631000

1.965737000

$-1.947525000$

$-3.128997000$

$-4.404678000$

$-2.975267000$

$-5.493667000$

$-4.066792000$

$-5.333187000$

2.736848000

1.780697000

2.276476000

$-4.544189000$

$-1.995067000$

$-6.472610000$

$-3.926137000$

$-6.183640000$

$-1.414084000$

$-0.549105000$

$-2.478600000$

$-1.176217000$

0.376256000

$-0.824219000$

$-0.261465000$

$-2.034986000$

$-1.090495000$
\end{abstract}

TSh: $E=-553.47724$

$-0.961565000$

$-2.462201000$

$-2.008277000$

$-0.464901000$

$-0.891592000$

0.179827000

$-0.735772000$

1.573512000

2.142256000

2.193236000

3. 586387000

3. 905876000

4.158108000

3. 718070000

$-2.237856000$

$-1.690262000$

$-1.899608000$

$-3.307781000$

$-1.374602000$

$-1.911368000$

$-2.102750000$

$-0.621765000$
$-1.158588000$

$-0.938357000$

$-2.203634000$

$-3.269086000$

$-0.176660000$

$-0.028767000$

1. 424712000

2.572668000

0.115588000

0.368174000

0.237234000

$-0.018165000$

0.462016000

$-0.349875000$

0.184348000

$-1.752406000$

$-0.663594000$

$-2.590083000$

$-2.050634000$

0.666462000

0.159557000

$-1.054542000$

1.257575000

$-2.175953000$

$-0.232845000$

$-3.667547000$

$-2.705657000$

2.889288000

3. 046047000

2.135992000

3.263517000

3. 567897000

2. 747206000

3. 854181000

3. 842523000

2. 345734000

$-1.273899000$

0.233565000

$-0.986913000$

$-2.226369000$

$-0.791066000$

0.476737000

1. 293779000

0.144437000

0.036760000

$-0.073624000$

$-0.425599000$

$-0.571907000$

0.379520000

$-1.344006000$

$-1.675608000$

$-1.147484000$

$-2.712014000$

$-1.645145000$

2.572026000

2.504889000

2. 850929000

3. 366200000
$-2.664306000$

1. 254759000

$-0.028797000$

0.191488000

$-1.667886000$

$-2.543496000$

$-0.412220000$

$-0.418404000$

1. 207111000

0.851090000

2. 547089000

3. 189512000

0.488726000

0.230693000

$-0.024010000$

0.261641000

$-0.239465000$

0.045916000

$-0.207118000$

2. 827292000

4. 250845000

3.038375000

$-0.052869000$

0.458497000

$-0.434264000$

0.072623000

$-0.376990000$

$-0.024375000$

0.931095000

0.179911000

$-1.425894000$

0.720740000

1.932602000

$-1.496859000$

$-1.771527000$

$-2.012243000$

0.995318000

0.302040000

0.235317000

0.842606000

1. 959992000

0.189280000

$-0.045111000$

0.359963000

1. 434412000

$-0.830830000$

$-0.748569000$

$-1.781177000$

$-0.278956000$

$-0.169425000$

$-1.040315000$

$-1.828915000$

$-0.970688000$

$-1.252338000$

$-0.379516000$

$-1.332467000$

0.390292000

$-0.457421000$

TSi: $E=-2176.77019$ 


\begin{abstract}
$-2.340286000$
$-3.208147000$

$-3.341732000$

$-4.564918000$

$-2.446642000$

$-1.468618000$

$-4.527328000$

$-4.627982000$

$-5.418151000$

$-1.237184000$

$-1.951461000$

$-0.224633000$

0.976845000

$-0.548194000$

0.500644000

$-2.224989000$

1.970441000

1.097969000

1.748924000

3.941664000

0.013014000

1.306042000

0.896636000

$-3.207307000$

$-2.206730000$

$-1.449081000$
\end{abstract}

TS2: $E=-1131.19818$
$-0.252225000$

$-1.139989000$

$-0.405136000$

0.406359000

0.435910000

$-0.886895000$

1.118476000

0.929957000

$-0.262183000$

0.456957000

$-0.411121000$

1. 375639000

1.100443000

2.662165000

3.643779000

$-1.194165000$

$-0.433018000$

$-1.362405000$

$-1.619885000$

0.307123000

4. 601334000

3. 476100000

3.593929000

$-0.949632000$

$-2.265755000$

$-0.980494000$

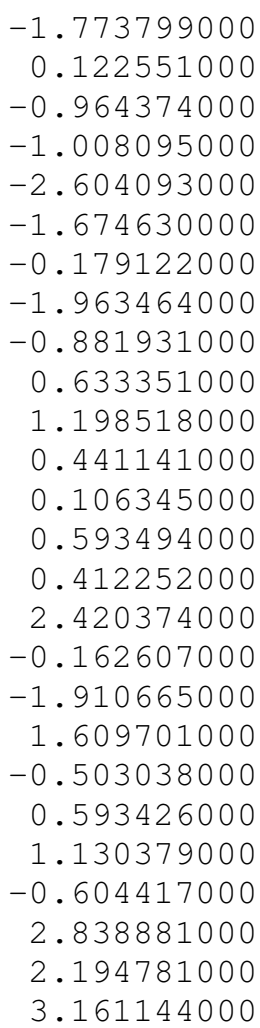

3.161144000
1.156262000

$-0.161510000$

0.541070000

1. 655185000

2.706772000

$-0.866016000$

$-1.299163000$

1. 359164000

2. 271184000

$-1.104661000$

$-1.717438000$

$-1.658922000$

$-2.568052000$

$-0.303953000$

$-1.111394000$

$-2.295603000$

2. 433976000

3. 572792000

2. 927075000

$-3.129727000$

$-2.064580000$

$-2.632028000$

0.986734000

0.551437000

$-0.772584000$

1. 523093000

$-1.106142000$

$-1.399591000$

1.078479000

2.391411000

1. 821502000
-1.710077000
0.146781000
-0.834143000
-1.529785000
-1.660848000
1.052286000
1.610075000
1.301111000
2.009186000
1.374772000
2.126136000
-1.030873000
-1.742492000
-0.885475000
-0.882782000
-1.526605000
-2.404327000
-2.009298000
-0.702355000
-0.820234000
-1.910539000
-2.363498000
1.201764000
0.925291000
0.759109000
0.351263000
0.527032000
1.393693000
0.268421000
1.011581000
-0.633620000 


\section{Cycloadducts 4-s-trans (from pathway B): 4a-i}

4a-s-trans: $\mathrm{E}=-1091.98399$

$\begin{array}{lrrr}6 & 2.010505000 & 0.506181000 & 1.550441000 \\ 24 & 1.254111000 & -0.294710000 & -0.003627000 \\ 6 & 2.671063000 & -1.535210000 & -0.054092000 \\ 8 & 3.539034000 & -2.299044000 & -0.085658000 \\ 8 & 2.483958000 & 0.951995000 & 2.505903000 \\ 6 & 0.358171000 & -1.512204000 & 1.154722000 \\ 8 & -0.101791000 & -2.288460000 & 1.877497000 \\ 6 & 0.505083000 & -1.097185000 & -1.562513000 \\ 8 & 0.085816000 & -1.588989000 & -2.519607000 \\ 6 & 2.306210000 & 0.809125000 & -1.147000000 \\ 8 & 3.002101000 & 1.404144000 & -1.852519000 \\ 6 & -0.391187000 & 1.035728000 & 0.035752000 \\ 6 & -1.780499000 & 0.642524000 & -0.013568000 \\ 8 & -0.377013000 & 2.375724000 & 0.104675000 \\ 6 & 0.810191000 & 3.176503000 & 0.088064000 \\ 7 & -3.790921000 & -0.488687000 & -0.452000000 \\ 6 & -2.392927000 & -0.750481000 & -0.031805000 \\ 6 & -2.845340000 & 1.498198000 & 0.026144000 \\ 8 & -4.037555000 & 0.928408000 & -0.070049000 \\ 6 & -4.794877000 & -1.275682000 & 0.248405000 \\ 1 & 1.219001000 & 3.216887000 & -0.923835000 \\ 1 & 0.483925000 & 4.173019000 & 0.391803000 \\ 1 & 1.556402000 & 2.801192000 & 0.787488000 \\ 1 & -1.949533000 & -1.431164000 & -0.760220000 \\ 1 & -2.353851000 & -1.221602000 & 0.964753000 \\ 1 & -2.862523000 & 2.576004000 & 0.124653000 \\ 1 & -5.789969000 & -0.943628000 & -0.057076000 \\ 1 & -4.660814000 & -2.314372000 & -0.070267000 \\ 1 & -4.699616000 & -1.217679000 & 1.344522000\end{array}$

4b-s-trans: $E=-1091.99157$

$\begin{array}{lr}6 & 1.693018000 \\ 24 & 1.258532000 \\ 6 & 2.978456000 \\ 8 & 4.035491000 \\ 8 & 1.980282000 \\ 6 & 0.752232000 \\ 8 & 0.546837000 \\ 6 & 0.825467000 \\ 8 & 0.603988000 \\ 6 & 1.973557000 \\ 8 & 2.492856000 \\ 6 & -0.725817000 \\ 6 & -1.937462000 \\ 8 & -1.085066000 \\ 6 & -0.168875000 \\ 7 & -3.470361000 \\ 6 & -2.050747000 \\ 6 & -3.233206000 \\ 8 & -4.154411000 \\ 6 & -3.824179000 \\ 1 & 0.227977000 \\ 1 & -0.765361000 \\ 1 & 0.641590000 \\ 1 & -3.917166000 \\ 1 & -1.433549000 \\ 1 & -1.814579000 \\ 1 & -3.669921000\end{array}$

0.949338000

$-0.149833000$

$-0.907880000$

$-1.377819000$

1.586136000

$-1.495633000$

$-2.320114000$

$-1.237345000$

$-1.887421000$

1. 127308000

1.842913000

0.640215000

$-0.135091000$

1. 934131000

3.029932000

$-1.870293000$

$-1.655219000$

0.339182000

$-0.626155000$

1. 701910000

3. 094390000

3. 918774000

2. 942746000

$-2.545218000$

$-2.153059000$

$-2.072419000$

2. 278025000
1.503181000

0.011599000

0.060518000

0.090329000

2. 424627000

1. 259196000

2. 043907000

$-1.492753000$

$-2.421538000$

$-1.207615000$

$-1.952932000$

$-0.040343000$

$-0.017496000$

$-0.084686000$

$-0.183843000$

$-0.375779000$

$-0.001694000$

0.061906000

0.012988000

0.207439000

$-1.199035000$

0.032698000

0.538581000

0.244566000

$-0.750200000$

0.987071000

$-0.711704000$ 
4c-s-trans: $E=-1073.56335$

$\begin{array}{lr}6 & 1.495676000 \\ 74 & 0.977146000 \\ 6 & 2.862626000 \\ 8 & 3.931959000 \\ 8 & 1.828134000 \\ 6 & 0.464323000 \\ 8 & 0.261035000 \\ 6 & 0.561517000 \\ 8 & 0.380919000 \\ 6 & 1.690591000 \\ 8 & 2.165672000 \\ 6 & -1.131222000 \\ 6 & -2.318225000 \\ 8 & -1.520566000 \\ 6 & -0.610293000 \\ 7 & -3.793866000 \\ 6 & -2.372234000 \\ 6 & -3.628150000 \\ 8 & -4.515958000 \\ 6 & -4.262511000 \\ 1 & -0.132413000 \\ 1 & -1.229146000 \\ 1 & 0.141246000 \\ 1 & -4.200836000 \\ 1 & -1.756876000 \\ 1 & -2.084305000 \\ 1 & -4.113767000 \\ 1 & -3.803149000 \\ 1 & -5.334643000\end{array}$

1.154554000

$-0.095140000$

$-0.873576000$

$-1.317747000$

1.832349000

$-1.509209000$

$-2.314783000$

$-1.370983000$

$-2.089947000$

1.259377000

1.981668000

0.693203000

$-0.116107000$

1. 981171000

3. 082199000

$-1.913411000$

$-1.637725000$

0.314196000

$-0.683010000$

1.658944000

3.104080000

3. 972962000

3. 036864000

$-2.581802000$

$-2.104121000$

$-2.048332000$

2. 234136000

2. 227906000

1. 545703000
1.555274000

0.007176000

0.068144000

0.103644000

2. 432856000

1. 408444000

2. 215067000

$-1.555331000$

$-2.442982000$

$-1.365705000$

$-2.135748000$

$-0.049689000$

$-0.024908000$

$-0.096426000$

$-0.180219000$

$-0.337361000$

$-0.009397000$

0.055076000

0.022983000

0.183787000

$-1.161695000$

$-0.053441000$

0.608400000

0.316385000

$-0.779893000$

0.968364000

$-0.736698000$

0.996097000

0.364950000

4d-s-trans: $E=-1131.27981$

$\begin{array}{lr}24 & 1.434577000 \\ 6 & 2.994571000 \\ 8 & 3.954126000 \\ 8 & 2.411404000 \\ 6 & 0.677744000 \\ 8 & 0.313964000 \\ 6 & 0.834182000 \\ 8 & 0.510256000 \\ 6 & 2.375488000 \\ 8 & 3.018974000 \\ 6 & -0.376986000 \\ 6 & -1.705867000 \\ 8 & -0.497273000 \\ 6 & 0.601360000 \\ 7 & -3.502567000 \\ 6 & -2.095807000 \\ 6 & -2.899884000 \\ 8 & -3.971606000 \\ 6 & -3.241738000 \\ 1 & 1.013770000 \\ 1 & 0.171939000 \\ 1 & 1.376577000 \\ 1 & -1.538848000 \\ 1 & -2.006007000 \\ 1 & -2.978035000 \\ 1 & -2.674922000\end{array}$

$-0.262602000$

$-1.309071000$

$-1.955357000$

1.282735000

$-1.518773000$

$-2.310052000$

$-1.240143000$

$-1.834281000$

0.883588000

1.505049000

0.871996000

0.326151000

2. 210814000

3. 125202000

$-1.100908000$

$-1.144672000$

1.019920000

0.229880000

2. 461684000

3.127345000

4.104626000

2.888599000

$-1.754701000$

$-1.596588000$

3. 023275000

2. 897403000
0.011581000

0.067958000

0.102233000

2. 464351000

1. 225822000

1. 986690000

$-1.510277000$

$-2.446731000$

$-1.183472000$

$-1.916482000$

$-0.042968000$

$-0.044548000$

$-0.062211000$

$-0.141749000$

$-0.500221000$

$-0.054067000$

0.034841000

$-0.053930000$

0.212655000

$-1.152747000$

0.079884000

0.586156000

$-0.765368000$

0.948835000

$-0.690733000$

1.039190000 


$\begin{array}{ll}1 & -4.314438000 \\ 6 & -4.377136000 \\ 1 & -5.407890000 \\ 1 & -4.070357000 \\ 1 & -4.311609000\end{array}$

2.566652000

$-2.074650000$

$-1.893119000$

$-3.060209000$

$-2.058158000$
0.396256000

0.135589000

$-0.178519000$

$-0.228904000$

1. 235374000

4e-s-trans: $E=-1112.85121$

$\begin{array}{lr}6 & 1.796330000 \\ 74 & 1.129822000 \\ 6 & 2.862776000 \\ 8 & 3.845900000 \\ 8 & 2.203321000 \\ 6 & 0.363564000 \\ 8 & 0.017397000 \\ 6 & 0.538439000 \\ 8 & 0.248740000 \\ 6 & 2.089633000 \\ 8 & 2.697527000 \\ 6 & -0.821004000 \\ 6 & -2.123620000 \\ 8 & -0.990270000 \\ 6 & 0.091751000 \\ 7 & -3.855762000 \\ 6 & -2.437100000 \\ 6 & -3.347922000 \\ 8 & -4.381561000 \\ 6 & -3.754607000 \\ 1 & 0.602326000 \\ 1 & -0.375251000 \\ 1 & 0.796361000 \\ 1 & -1.864872000 \\ 1 & -2.294591000 \\ 1 & -3.515901000 \\ 1 & -3.207709000 \\ 1 & -4.830781000 \\ 6 & -4.666171000 \\ 1 & -5.711653000 \\ 1 & -4.319261000 \\ 1 & -4.576004000 \\ & \\ 8 & \\ 6 & \\ 6 & -1000\end{array}$

0.933603000

$-0.167514000$

$-1.242195000$

$-1.854178000$

1.523476000

$-1.532948000$

$-2.329810000$

$-1.302479000$

$-1.950831000$

1.092273000

1. 746782000

0.958664000

0.356978000

2. 294798000

3.230345000

$-1.161485000$

$-1.130618000$

0.993328000

0.152243000

2. 418774000

3.187235000

4.209637000

3.049512000

$-1.704859000$

$-1.582148000$

2.989340000

2.881881000

2.476265000

$-2.164875000$

$-2.040258000$

$-3.140957000$

$-2.124126000$
1. 609725000

0.006352000

0.073545000

0.112105000

2.518902000

1. 339867000

2.105249000

$-1.605823000$

$-2.518857000$

$-1.304461000$

$-2.041349000$

$-0.057609000$

$-0.067585000$

$-0.076364000$

$-0.108645000$

$-0.488450000$

$-0.077823000$

0.017569000

$-0.057258000$

0.190143000

$-1.072745000$

0.015849000

0.703805000

$-0.807364000$

0.919185000

$-0.714526000$

1. 015318000

0.374097000

0.185699000

$-0.106590000$

$-0.168118000$

1. 283131000

4f-s-trans: $E=-1382.66467$

$\begin{array}{lr}6 & 2.090284000 \\ 24 & 1.626553000 \\ 6 & 3.324117000 \\ 8 & 4.364875000 \\ 8 & 2.394819000 \\ 6 & 1.062372000 \\ 8 & 0.816327000 \\ 6 & 1.166478000 \\ 8 & 0.923312000 \\ 6 & 2.384367000 \\ 8 & 2.922729000 \\ 6 & -0.318430000 \\ 6 & -1.554983000 \\ 8 & -0.653981000 \\ 6 & 0.280821000 \\ 7 & -3.155515000 \\ 6 & -1.754387000 \\ 6 & -2.818928000 \\ 8 & -3.788721000 \\ 1 & 0.692798000\end{array}$

0.865137000

$-0.183831000$

$-0.999254000$

$-1.503391000$

1.469780000

$-1.544860000$

$-2.383764000$

$-1.226401000$

$-1.852754000$

1.104329000

1.827698000

0.655377000

$-0.082086000$

1.959814000

3. 044692000

$-1.737432000$

$-1.590971000$

0.468631000

$-0.444992000$

3. 138210000
1.530753000

0.009841000

0.048686000

0.071368000

2. 467567000

1. 217575000

1.973843000

$-1.518864000$

$-2.458069000$

$-1.172439000$

$-1.895838000$

$-0.043427000$

$-0.067491000$

$-0.055642000$

$-0.102018000$

$-0.484608000$

$-0.043973000$

$-0.029501000$

$-0.110411000$

$-1.108677000$ 

3.934366000
2. 914260000
$-2.140457000$
$-2.000880000$
$-2.774612000$
$-2.723672000$
$-3.735708000$
$-2.701237000$
2.263676000
2.180035000
3.023148000
2.895412000

$$
\begin{array}{r}
0.140015000 \\
0.628792000 \\
-0.743567000 \\
0.969435000 \\
0.201110000 \\
-0.111480000 \\
-0.121535000 \\
1.298592000 \\
0.119221000 \\
0.234861000 \\
-1.099505000 \\
1.335028000
\end{array}
$$

\begin{tabular}{|c|c|}
\hline 6 & -2.092926000 \\
\hline 24 & -2.197813000 \\
\hline 6 & -4.022778000 \\
\hline 8 & -5.145984000 \\
\hline 8 & -2.121360000 \\
\hline 6 & -1.791356000 \\
\hline 8 & -1.576574000 \\
\hline 6 & -2.521108000 \\
\hline 8 & -2.823200000 \\
\hline 6 & -2.611843000 \\
\hline 8 & -2.905914000 \\
\hline 6 & -0.114936000 \\
\hline 6 & 0.762473000 \\
\hline 8 & 0.623438000 \\
\hline 6 & 0.184991000 \\
\hline 7 & 1.587302000 \\
\hline 6 & 0.323146000 \\
\hline 6 & 2.134768000 \\
\hline 8 & 2.640022000 \\
\hline 1 & -0.772746000 \\
\hline 1 & 0.960563000 \\
\hline 1 & 0.122903000 \\
\hline 1 & -0.355507000 \\
\hline 1 & -0.146112000 \\
\hline 6 & 1.744997000 \\
\hline 1 & 2.743612000 \\
\hline 1 & 1.005934000 \\
\hline 1 & 1.583354000 \\
\hline 6 & 3.176123000 \\
\hline 6 & 3.941297000 \\
\hline 6 & 3.474519000 \\
\hline 1 & 3.722227000 \\
\hline 1 & 2.896604000 \\
\hline 6 & 4.968705000 \\
\hline 6 & 4.516528000 \\
\hline 1 & 5.544066000 \\
\hline 1 & 4.748608000 \\
\hline 6 & 5.259776000 \\
\hline 1 & 6.06520800 \\
\hline
\end{tabular}

\section{4g-s-trans: $E=-1322.96156$}

$-1.748509000$

$-0.249541000$

$-0.167297000$

$-0.115763000$

$-2.632370000$

0.861523000

1. 510169000

1.242564000

2.122403000

$-1.358235000$

$-2.003499000$

$-0.299402000$

0.844836000

$-1.379568000$

$-2.733844000$

3.036359000

2. 302333000

0.862791000

2.103880000

$-2.901371000$

$-3.336651000$

$-2.988724000$

2. 561760000

2. 585590000

4.240943000

4.653396000

4.962769000

4.066179000

$-0.168321000$

$-0.126354000$

$-1.122214000$

0.620703000

$-1.143922000$

$-1.049814000$

$-2.028999000$

$-1.021163000$

$-2.757942000$

$-2.000993000$

$-2.714221000$
1.267296000

0.095946000

0.538153000

0.812237000

2. 012459000

1. 589542000

2. 521780000

$-1.040033000$

$-1.726948000$

$-1.397201000$

$-2.310065000$

$-0.387123000$

$-0.447270000$

$-0.659470000$

$-0.504783000$

$-0.660619000$

$-0.436498000$

$-0.278143000$

$-0.215684000$

$-0.995786000$

$-0.980879000$

0.554985000

$-1.249636000$

0.520314000

0.141532000

$-0.022002000$

$-0.220623000$

1. 217472000

$-0.065200000$

1.111745000

$-1.050179000$

1. 869173000

$-1.967798000$

1. 314599000

$-0.852011000$

2. 235968000

$-1.624038000$

0.332723000

0.487114000

4h: $E=-553.57191$

0.067632000

$-0.699392000$

$-2.068043000$

$-2.111843000$

$-0.833593000$

$-0.675683000$
0.072668000

$-1.229870000$

$-0.783695000$

0.654138000

1. 084475000

2.560902000
$-0.078883000$ $-0.100139000$ $-0.454252000$ $-0.082690000$ $-0.022897000$ 0.095709000 
$-3.127751000$

1.515058000

2. 161748000

2.105365000

3.541059000

$-0.665666000$

$-0.353077000$

$-1.084302000$

0.379247000

$-1.237042000$

$-4.085791000$

$-2.969156000$

$-3.151333000$

3. 827725000

3.886442000

3. 963116000

4i: $E=-2176.85492$

3. 143175000

4.115268000

3.354009000

2.056151000

1. 157093000

5.245401000

0.561683000

$-0.554683000$

0.645081000

$-0.572436000$

3.256359000

3. 305611000

1. 748917000

0.451216000

0.563129000

5.844816000

4.940700000

5.852743000

$-0.232718000$

$-1.219876000$

$-1.097968000$

$-2.143345000$

$-3.628228000$

$-1.678092000$

$-2.480006000$

5: $E=-1131.26837$

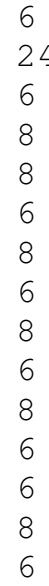

1.989157000

1. 333085000

2. 768932000

3.646312000

2.404548000

0.342454000

$-0.176330000$

0.703980000

0.402405000

2.431144000

3.156007000

$-0.308194000$

$-1.693541000$

$-0.390668000$

0.756449000
$-1.423121000$

0.200268000

1. 238260000

$-1.029424000$

$-1.031977000$

$-1.739410000$

$-1.934195000$

3. 053320000

2. 815644000

2.930186000

$-0.964928000$

$-1.363807000$

$-2.475035000$

$-2.084410000$

$-0.534214000$

$-0.521146000$

0.376082000

1.080309000

0.005915000

$-1.256786000$

$-0.978181000$

$-2.162627000$

$-0.079615000$

1.029752000

0.458565000

2. 353100000

3.138874000

1. 461768000

1. 908366000

$-3.073140000$

$-2.076682000$

$-2.230580000$

$-0.957517000$

$-0.122879000$

0.815845000

4.173222000

2. 915324000

2. 924833000

$-0.321196000$

1. 219727000

$-1.042906000$

$-1.851587000$

0.836175000

$-0.252594000$

$-1.453497000$

$-2.186256000$

1. 470717000

$-1.261709000$

$-1.887398000$

$-1.387844000$

$-2.084929000$

0.653324000

1.140995000

0.979148000

0.487872000

2. 290537000

3. 153462000

\begin{abstract}
0.309967000
$-0.014632000$

0.019272000

0.006007000

0.068139000

0.881952000

$-0.862363000$

$-0.795120000$

0.200655000

0.962444000

0.052193000

1.399582000

0.006484000

0.072825000

0.978560000

$-0.801753000$
\end{abstract}

-0.240052000
-0.047661000
-0.355830000
-0.184902000
-0.246820000
-0.300622000
0.559329000
-0.297170000
-0.451900000
-0.215576000
-0.292713000
0.983326000
-0.743583000
-0.416597000
-1.132330000
0.617757000
0.308266000
1.616810000
0.393512000
-0.263228000
0.556083000
-1.224954000
0.081460000
0.070870000
2.051073000
-1.371940000

1.462032000

0.051956000

0.328294000

0.498809000

2.335705000

1. 349879000

2. 167248000

$-1.371648000$

$-2.236369000$

$-1.207787000$

$-1.965978000$

$-0.231792000$

$-0.185357000$

$-0.430332000$

$-0.509858000$ 


$\begin{array}{rrr}-3.302020000 & -1.066184000 & -0.567797000 \\ -1.853614000 & -0.869437000 & -0.366329000 \\ -2.864109000 & 1.113544000 & 0.104466000 \\ -3.919865000 & 0.037458000 & 0.179672000 \\ -3.604194000 & -2.396791000 & -0.063357000 \\ -3.184425000 & 2.534669000 & 0.429826000 \\ 1.270825000 & 2.991852000 & -1.458477000 \\ 0.350796000 & 4.165877000 & -0.475620000 \\ 1.433466000 & 2.988452000 & 0.327683000 \\ -4.139466000 & -0.215387000 & 1.240348000 \\ -4.863873000 & 0.316052000 & -0.304167000 \\ -4.667549000 & -2.579562000 & -0.251345000 \\ -3.021002000 & -3.132785000 & -0.621951000 \\ -3.398365000 & -2.502936000 & 1.014101000 \\ -3.640657000 & 3.040017000 & -0.434253000 \\ -2.301660000 & 3.107245000 & 0.717379000 \\ -3.918443000 & 2.578019000 & 1.245691000\end{array}$

